\title{
Ablation-cooled material removal with ultrafast bursts of pulses
}

\author{
Can Kerse ${ }^{1}$, Hamit Kalaycıoğlu², Parviz Elahi², Barbaros Çetin ${ }^{3}$, Denizhan K. Kesim¹ ${ }^{1}$ Önder Akçaalan ${ }^{1}$, Seydi Yavaş ${ }^{4}$, \\ Mehmet D. Aşık ${ }^{5}$, Bülent Öktem ${ }^{6}$, Heinar Hoogland ${ }^{7,8}$, Ronald Holzwarth ${ }^{7} \&$ Fatih Ömer Ilday ${ }^{1,2}$
}

\begin{abstract}
The use of femtosecond laser pulses allows precise and thermaldamage-free removal of material (ablation) with wide-ranging scientific $^{1-5}$, medical ${ }^{6-11}$ and industrial applications ${ }^{12}$. However, its potential is limited by the low speeds at which material can be removed ${ }^{1,9-11,13}$ and the complexity of the associated laser technology. The complexity of the laser design arises from the need to overcome the high pulse energy threshold for efficient ablation. However, the use of more powerful lasers to increase the ablation rate results in unwanted effects such as shielding, saturation and collateral damage from heat accumulation at higher laser powers ${ }^{6,13,14}$. Here we circumvent this limitation by exploiting ablation cooling, in analogy to a technique routinely used in aerospace engineering ${ }^{15,16}$. We apply ultrafast successions (bursts) of laser pulses to ablate the target material before the residual heat deposited by previous pulses diffuses away from the processing region. Proof-of-principle experiments on various substrates demonstrate that extremely high repetition rates, which make ablation cooling possible, reduce the laser pulse energies needed for ablation and increase the efficiency of the removal process by an order of magnitude over previously used laser parameters ${ }^{17,18}$. We also demonstrate the removal of brain tissue at two cubic millimetres per minute and dentine at three cubic millimetres per minute without any thermal damage to the bulk ${ }^{9,11}$.
\end{abstract}

Ablation is the evaporative removal of a material when its temperature exceeds a critical value. Because the ablated material is physically carried away, the thermal energy contained in the ablated mass is also removed, thus reducing the average temperature of the remaining material. This effect forms the basis of ablation cooling, which has been routinely used as an approach to thermal protection during the atmospheric re-entry of rockets since the 1950s, owing to the minimal mass requirements ${ }^{15}$. Unlike ablation cooling for rockets, laser ablation is not continuous, but takes place only during and shortly after an incident laser pulse. For the laser parameters used in previous experiments ablation cooling has been negligible as a cooling mechanism in comparison with heat conduction (diffusion) from the processing region into the bulk of the target, which is continuously occurring. For ablation cooling to become a major contributor, the time delay between the laser pulses (the inverse of the repetition rate) must be reduced until the part of the material that is to be ablated does not cool substantially between successive pulses. Only then would heat extraction due to ablation become comparable to that due to diffusion (Fig. 1a).

The physics of the ablation-cooled regime can be explained through a toy model (see Supplementary Information section 1 for full details). We assume that each pulse gives rise to an instantaneous temperature rise of $\Delta T$, which is roughly proportional to the pulse energy, $E_{\mathrm{p}}$, and that the material cools with a $1 / \sqrt{1+t / \tau_{0}}$ dependence on the time delay, $t$, after the arrival of a pulse. The thermal relaxation time, $\tau_{0}$, is proportional to $\delta^{2} / \alpha$, where $\delta$ is the depth or the lateral radius (whichever dimension is smaller) of the section of the material to be ablated and $\alpha$ is its thermal diffusivity. For a train of $N$ pulses, the temperature of the target surface that is encountered by the $(n+1)$ th pulse is given by $T_{n+1}=T_{n}+\delta T$, where $\delta T=\Delta T / \sqrt{1+\tau_{\mathrm{R}} / \tau_{0}}$ is the small net increase in target temperature by a single pulse and $\tau_{\mathrm{R}}$ is inverse of the repetition rate. Ablation occurs when the temperature exceeds a critical value $T_{\mathrm{c}}$. For the traditional regime of ultrafast ablation, the repetition rate is low $\left(\tau_{\mathrm{R}} \gg \tau_{0}\right)$ and each pulse must be energetic enough to cause ablation $\left(\Delta T>T_{\mathrm{c}}-T_{0}\right.$, where $T_{0}$ is the initial surface temperature). The ablation-cooled regime corresponds to $\tau_{\mathrm{R}} \lesssim \tau_{0}$. In this regime, the energy of the individual pulses can be lower than the ablation threshold because temperature builds up from pulse to pulse and ablation starts after the $m$ th pulse in the train, where $m=\left(T_{\mathrm{c}}-T_{0}-\Delta T+\delta T\right) / \delta T$. The volume of the ablated material is given by $V_{\text {ablated }}=\beta[N-$ $\left.u\left(T_{\mathrm{c}}-T_{0}-\Delta T\right) m\right] E_{\mathrm{p}} u(N-m)$, where $\beta$ is a proportionality factor and $u$ is the Heaviside (unit step) function. The thermal energy that diffuses into the bulk of the target owing to cooling between the pulses is $E_{\text {heat }}=\alpha\left(T_{\mathrm{c}}-T_{0}\right)\left(1-\frac{1}{\sqrt{1+\tau_{\mathrm{R}} / \tau_{0}}}\right)(N-m) E_{\mathrm{p}}+\alpha(\Delta T-\delta T) m E_{\mathrm{p}}$. For the traditional regime, this result reduces to $\lim _{\tau_{\mathrm{R}} \rightarrow \infty} E_{\text {heat }}=\alpha\left(T_{\mathrm{c}}-T_{0}\right) N E_{\mathrm{p}}$. The toy model makes two main predictions for the ablation-cooled regime-both are confirmed by numerical solutions of the heat diffusion equation (see Supplementary Information section 2 for details) as well as the experiments described below. The first is that increasing the repetition rate reduces the heating of surrounding regions (Fig. 1b, c and Supplementary Fig. 1). Because less of the deposited laser energy is lost to heat diffusion $\left(\lim _{\tau_{\mathrm{R}} \rightarrow 0} E_{\text {heat }}=0\right)$, the ablation efficiency is higher than for the traditional regime (Supplementary Fig. 3). The second states that the pulse energy can be decreased if the number of pulses is simultaneously increased in proportion, without a subsequent reduction in the ablation efficiency (Fig. 1d). This is necessary to fully benefit from the ablation-cooled regime, because shielding effects (that is, ablation-induced plasma and ejected particulates reflecting and scattering incoming light) will prevent efficient ablation if the repetition rate is increased at a constant energy ${ }^{18}$.

To demonstrate ablation cooling, a customized femtosecond fibre laser ${ }^{19-21}$ was used (see Supplementary Information section 3 for details). We implemented burst-mode operation ${ }^{22}$, because continuous trains of energetic pulses at the high repetition rates required to access the ablation-cooled regime correspond to a prohibitively high average power and laser repositioning in continuous mode is limited. In burst mode, the laser produces groups of high-repetition-rate pulses, which are, in turn, repeated with a lower frequency. The duty cycle of the 
a

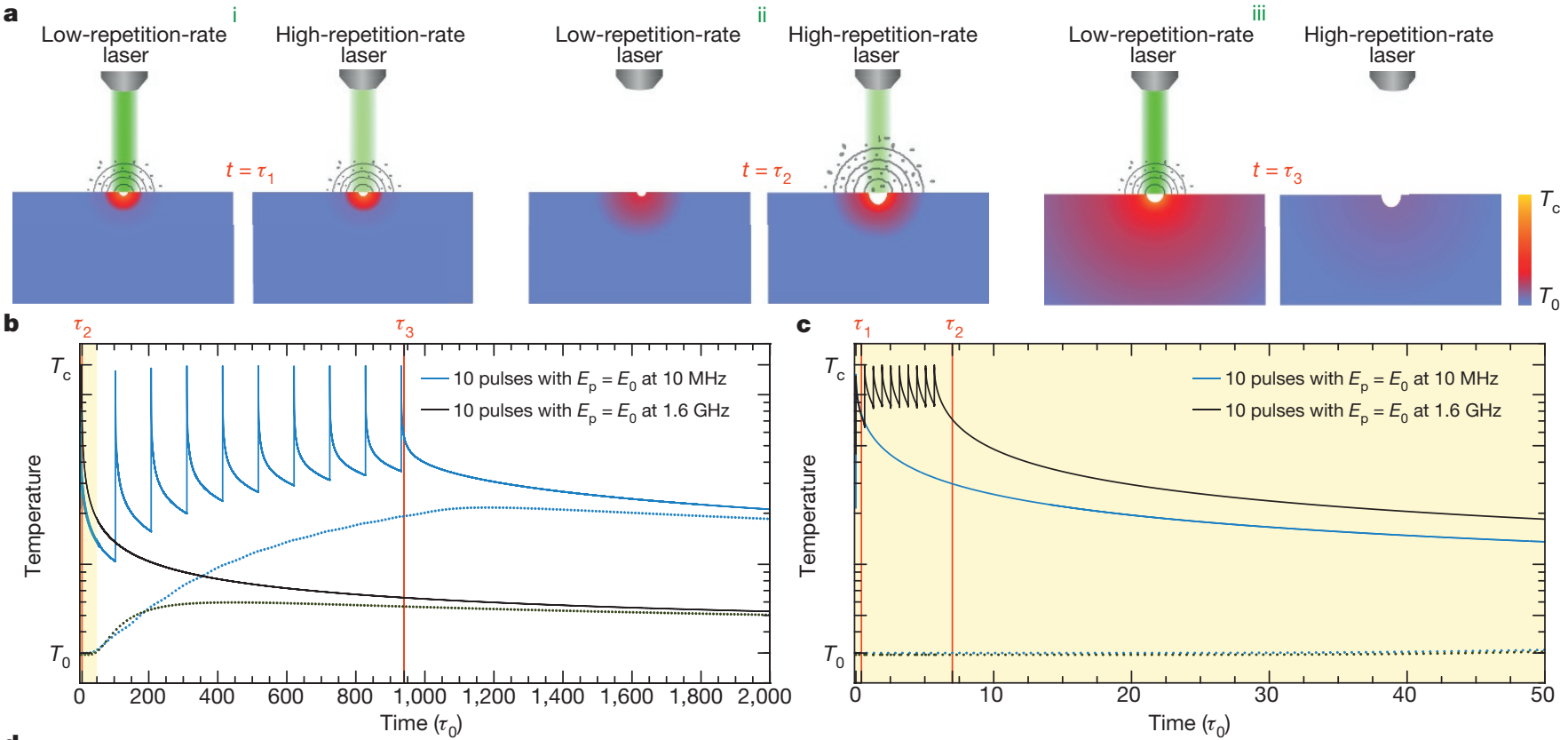

d
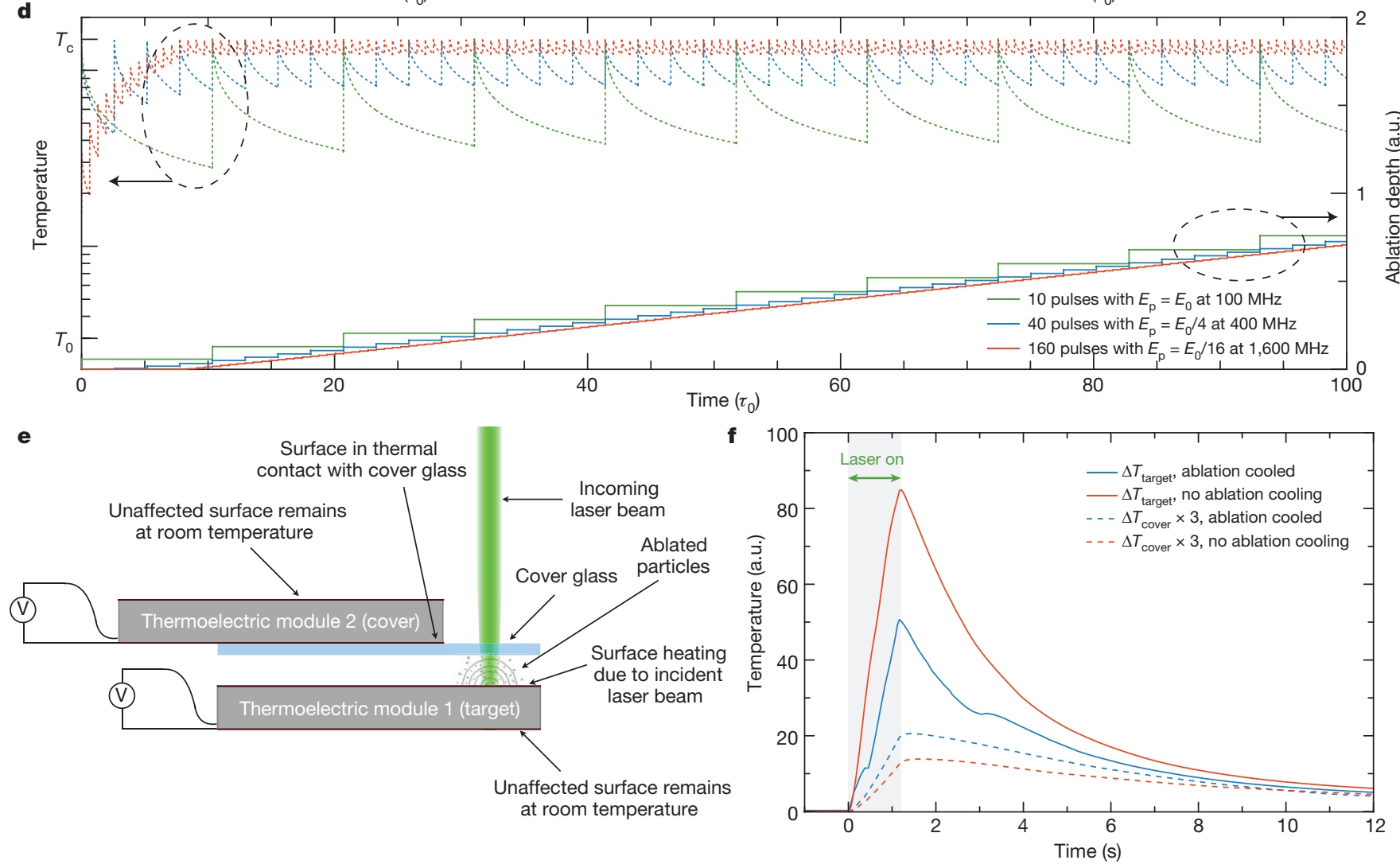

Figure 1 | Principles of ablation-cooled removal of a material by laser. a, Schematic representation of the ablation process for low (traditional regime, left diagrams) and high (ablation-cooled regime, right diagrams) repetition rates. Temperature profiles are illustrated for $t=\tau_{1}$ (i), which is shortly after the arrival of the first pulse for both cases; for $t=\tau_{2}$ (ii), which is before (shortly after) the arrival of the second (last) pulse for the low-repetition-rate (high-repetition-rate) laser; and for $t=\tau_{3}$ (iii), which is shortly after the arrival of the last pulse for the low-repetition-rate laser. The colouration of the target material is based on simulation results shown in $\mathbf{b}$ at the indicated time intervals of $\tau_{1}, \tau_{2}$ and $\tau_{3}$. $\mathbf{b}$, Calculated evolution of the temperatures at the surface (solid lines) and below (at a depth of 30 times the optical penetration depth) the surface (dotted lines) for repetition rates of $10 \mathrm{MHz}$ (blue lines) and $1,600 \mathrm{MHz}$ (black lines).

The pulse energies and number of pulses are the same for both cases.

The higher repetition rate results in substantially lower temperatures below the surface due to ablation cooling. c, Expanded view of the shaded section of the plot in $\mathbf{b}$. d. Calculated evolution of the surface temperature (dashed lines) and amount of ablated material (solid lines) for repetition rates of $100 \mathrm{MHz}$ (green lines), $400 \mathrm{MHz}$ (blue lines) and 1,600 MHz (red lines). The ablation rate remains approximately the same when the product of the pulse energy and repetition rate is maintained. The spikes in the surface temperatures precisely indicate the arrival of pulses, which are not shown explicitly for clarity. e, Experimental set-up for direct confirmation of the ablation-cooling effect. $\mathbf{f}$, The measured temperature increase that is induced on thermoelectric module 1 (the target material; solid lines) and thermoelectric module 2 (attached to the coverslip that collects a portion of the ablated particles; dashed lines, values have been multiplied by three to aid comparison with $\Delta T_{\text {target }}$ ) with the laser operating in the ablationcooled regime (blue lines) and in the traditional regime (red lines). 
pulsation can be adjusted to set the average power. Burst-mode material processing has substantial benefits ${ }^{22-24}$, but the possibility of ablation cooling has not yet been recognized.

First we present experimental evidence of the ablation-cooling effect by simultaneously measuring the temperature of a target material directly and the heat carried by the ablated particles (indirectly) (Fig. 1e). The laser beam is focused onto and ablates the surface of a thermoelectric module. This causes a temperature difference between the laser-targeted top surface and the bottom surface, which generates a voltage difference by the Seebeck effect. A portion of the particles ejected from the surface during ablation stick to a glass coverslip, which is held approximately $1 \mathrm{~mm}$ above the target. A second thermoelectric module is used to monitor the temperature of the coverslip, which rises in proportion to the thermal energy delivered by the ablated particles. The measured temperatures of the target and the coverslip (Fig. 1f) confirm that the target heats less, and the coverslip more, in the ablation-cooled regime. The laser parameters were 50 pulses of $3 \mu \mathrm{J}$ each, with an $800 \mathrm{fs}$ duration for a $0.2 \mathrm{MHz}$ burst and a $1.7 \mathrm{GHz}$ intraburst repetition rate. This is within the ablation-cooled regime assuming a typical thermal diffusivity of about $150 \mathrm{~mm}^{2} \mathrm{~s}^{-1}$ for the ceramic surface of the thermoelectric module and $3 \mu \mathrm{J}, 800 \mathrm{fs}$ pulses at a $10 \mathrm{MHz}$ uniform repetition rate to illustrate the traditional regime $(10 \mathrm{MHz}$ was chosen to be safely outside the ablation-cooled regime, although the thermal diffusivity of the ceramic surface is not precisely known).

We demonstrate validity of the predictions of the toy model for ablation cooling across a range of materials (see Supplementary Information for a discussion of other materials). Copper and silicon were chosen as examples of metal and semiconductor targets, respectively, because their ablation rates with ultrafast pulses are well documented. The volume of material ablated as a function of the incident energy is shown in Fig. 2a for $\mathrm{Cu}$ and Fig. $2 \mathrm{~b}$ for $\mathrm{Si}$ for various repetition rates. Figure $2 \mathrm{c}$, $\mathrm{d}$ shows the number of atoms ablated per incident photon as a function of the pulse energy. We observe a substantial increase in ablation when the repetition rate is about $100 \mathrm{MHz}$ or higher. Although it is not possible to predict the precise frequency required for each material (the toy model is too simple for us to expect quantitatively accurate predictions), $\tau_{0} \approx 1 \mathrm{~ns}$ for $\mathrm{Cu}$ for a processing region depth of a few hundred nanometres. Given that increases in efficiency are predicted to begin at a tenth of the corresponding repetition rate, this prediction agrees with the experimental observations. The lower thermal diffusivity of $\mathrm{Si}$ compared with $\mathrm{Cu}$ is consistent with the increase in its ablation efficiency at $27 \mathrm{MHz}$, whereas the results at $1 \mathrm{MHz}$ and $27 \mathrm{MHz}$ are similar for $\mathrm{Cu}$, implying that the onset of the ablation-cooled regime for $\mathrm{Cu}$ begins between $27 \mathrm{MHz}$ and $108 \mathrm{MHz}$. If the repetition rate is further increased, efficiency saturates at high pulse energies-a consequence of the expected shielding effect. The solution is to decrease the pulse energy, and increase the number of pulses and the repetition rate (for example, from 25 pulses at $108 \mathrm{MHz}$ to 800 pulses (with 32 times lower energy) at $3,464 \mathrm{MHz}$ ). The amount of ablation remains similar (black and pink data in Fig. 2a, b), which means that the shielding effects have been overcome.

To place the ablation results into context, they should be compared with common literature values (see Supplementary Information section 5 for an extensive discussion). Comparison with experiments on $\mathrm{Cu}$ using $70 \mathrm{fs}$ pulses with a pulse energy of up to $0.4 \mathrm{~mJ}$ at $800 \mathrm{~nm}$ (ref. 17) a

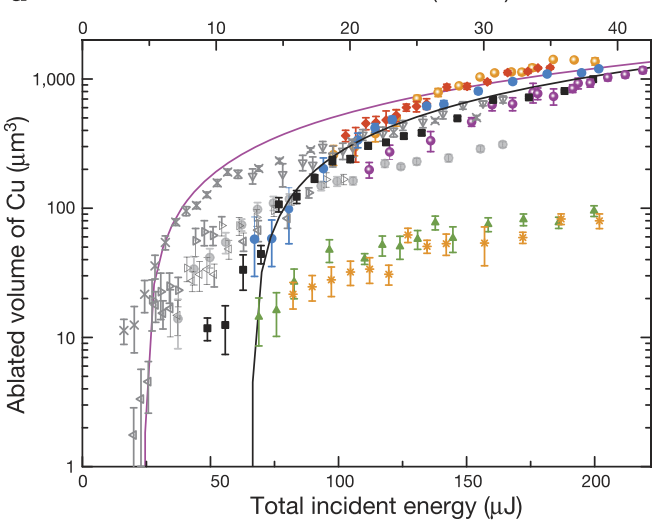

c

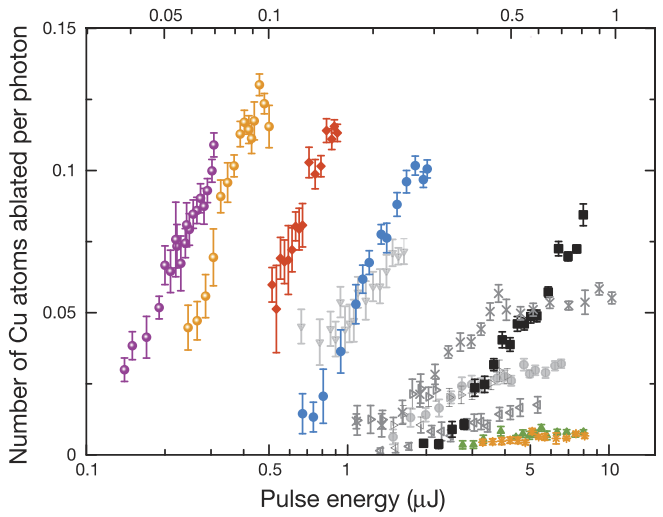

Figure 2 | Scaling down of the pulse energy with increasing repetition rate. a, b, Volumes (symbols) of $\mathrm{Cu}(\mathbf{a})$ and $\mathrm{Si}(\mathbf{b})$ ablated by a single burst of pulses as a function of total incident energy and fluence for different intraburst repetition rates. The predictions of the toy model for the lowest and highest repetition rates in the ablation-cooled regime are also shown (solid lines). c, d, Ablation efficiency in terms of number of atoms of $\mathrm{Cu}$ (c) and $\mathrm{Si}(\mathbf{d})$ ablated per incident photon as a function of pulse energy and
Total incident fluence $\left(\mathrm{J} \mathrm{cm}^{-2}\right)$
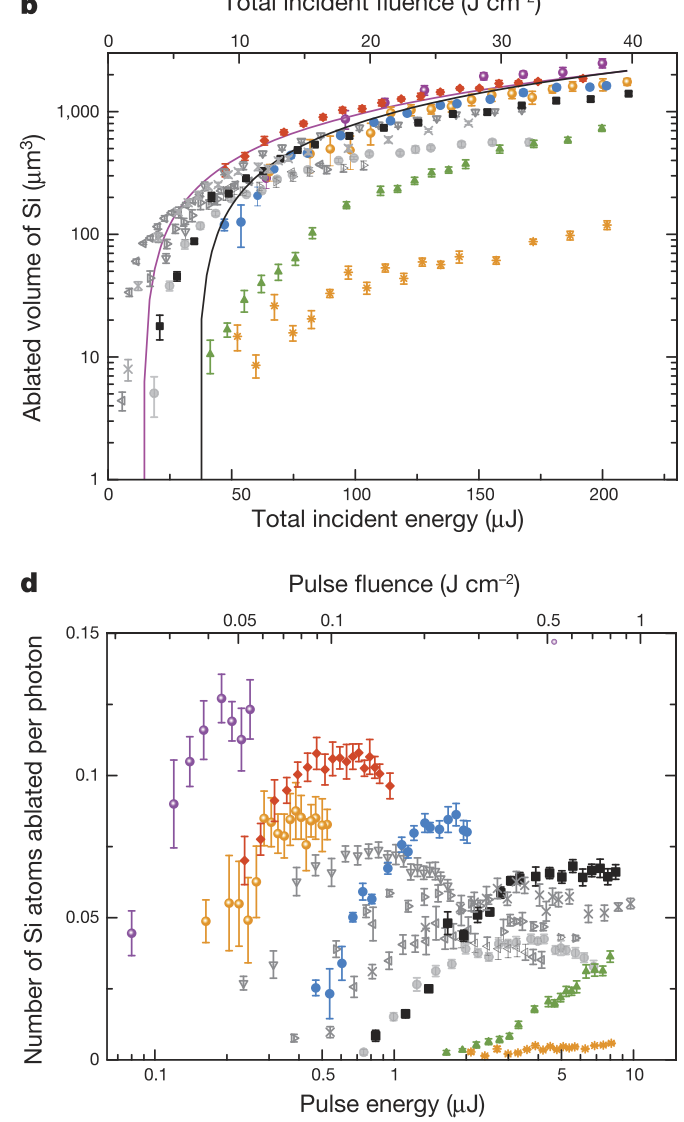

pulse fluence for different repetition rates. The legend applies to all panels. The lower and upper limits to the data correspond to the ablation threshold and available laser energy, respectively. In all panels the sample size for each data point is 20, where the centre values represent the mean and the error bars represent the standard deviation. Coloured symbols highlight the onset of the ablation-cooled regime and (beyond $108 \mathrm{MHz}$ ) the inverse scaling of the pulse energy with repetition rate in the ablation-cooled regime. 


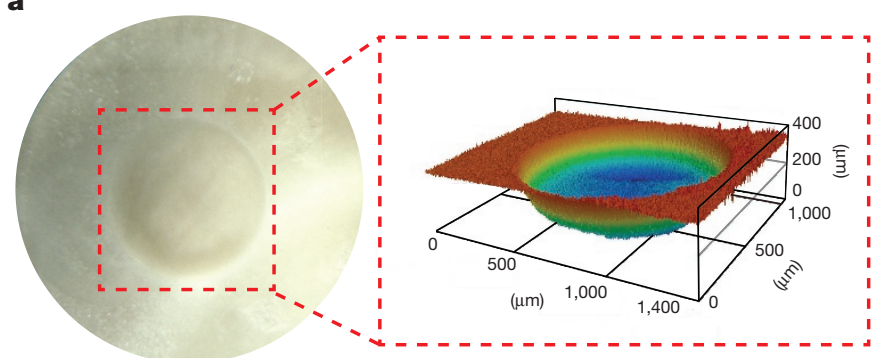

$0.5 \mathrm{~mm}$

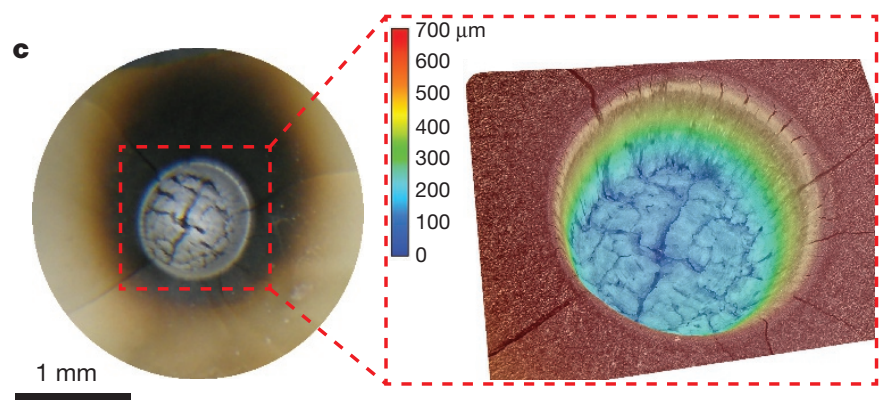

e

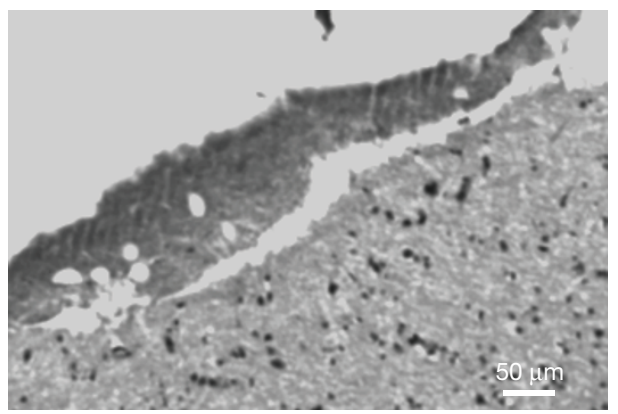

g

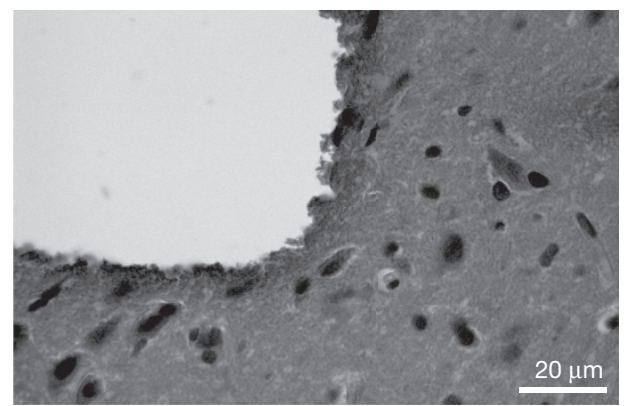

Figure 3 Ablation of hard and soft tissues. a, b, Laser removal of a section of human dentine obtained in the traditional regime $(\mathbf{a}, 1 \mathrm{kHz}$ uniform repetition rate) and in the ablation-cooled regime $(\mathbf{b}, 1.7 \mathrm{GHz}$ intraburst repetition rate). Although both ablation cooling and traditional ultrafast processing avoid thermal damage at sufficiently low average powers, the ablation-cooled regime achieves approximately six times more ablation despite using pulse energies that are about 12 times lower. c, d, When the (uniform or intraburst, respectively) repetition rate, average power and scanning speed are simultaneously increased by a factor of 25 , the traditional regime of ultrafast processing results in thermal damage (c; Supplementary Video 4), whereas the ablation-cooled regime completely avoids thermal effects and achieves an ablation speed of $3 \mathrm{~mm}^{3} \mathrm{~min}^{-1}$, despite using a pulse

reveals that we obtain around 2,000 times more ablation at the same fluence of approximately $0.04 \mathrm{~J} \mathrm{~cm}^{-2}$, which is our maximum fluence for the intraburst repetition rate of $3,456 \mathrm{MHz}$. Even if we imagine the entire burst of 800 pulses to act like a single pulse and compare the results with those in ref. 17 for an equal total fluence $\left(20 \mathrm{~J} \mathrm{~cm}^{-2}\right.$, the highest value for which direct comparison is possible), we obtain about 12 times more ablation, although our pulse fluence (energy) is
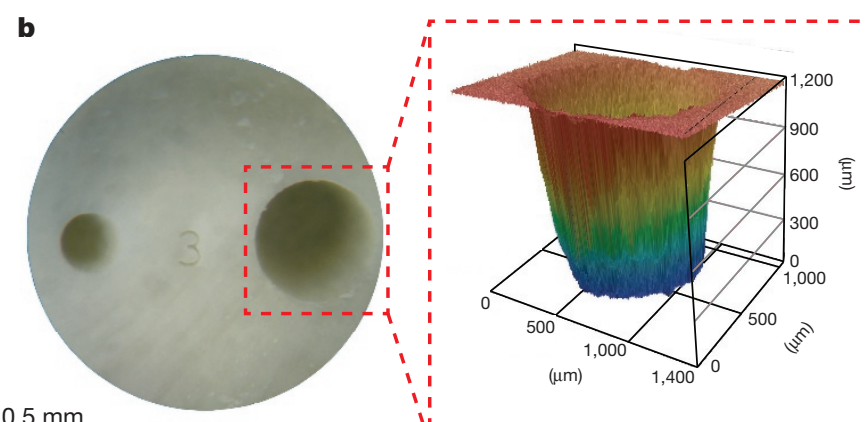

$0.5 \mathrm{~mm}$
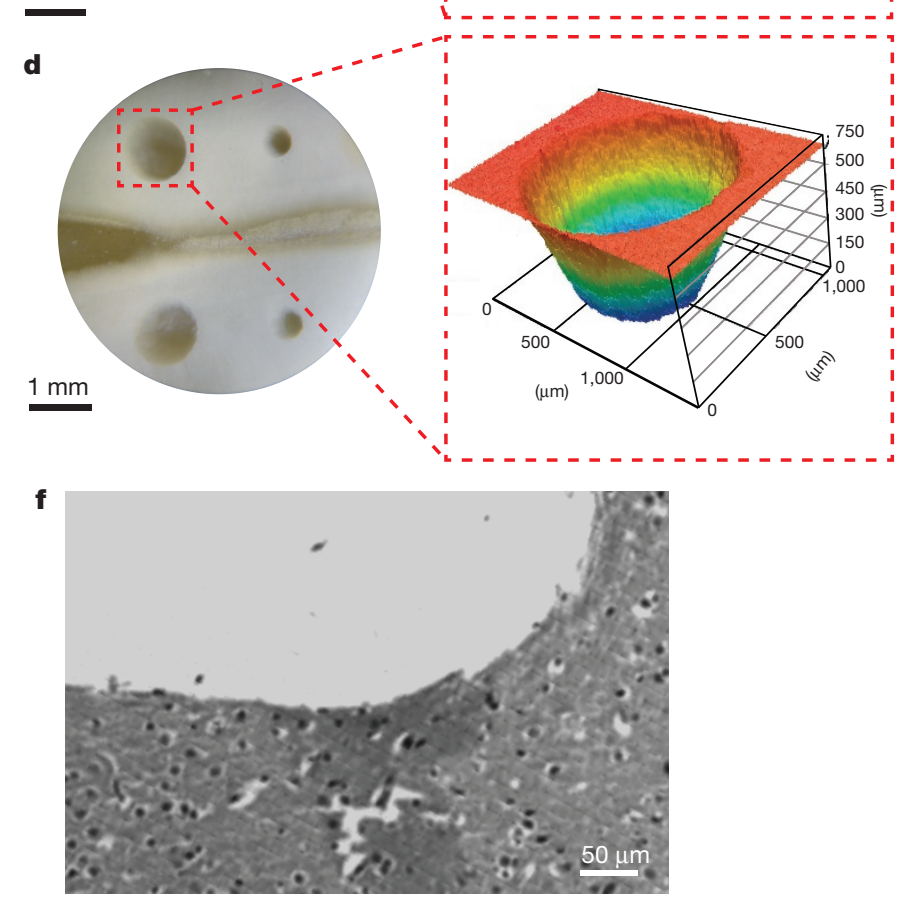

h

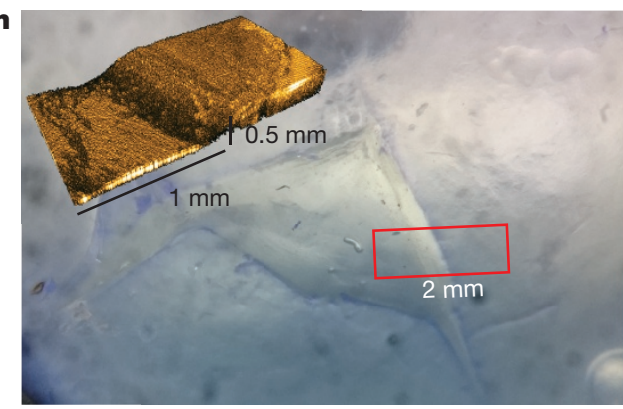

energy that is 25 times lower (d; Supplementary Video 5). The insets in a-d show laser scanning microscope characterizations of the ablated holes. e, f, Histological images corresponding to about $1 \mathrm{~mm}^{3}$ sections, which were removed from a rat brain with the laser operating at an average power of $600 \mathrm{~mW}$ in the traditional regime (e), showing presence of thermal damage, and in the ablation-cooled regime (f), showing no major thermal damage. g, Ablation-cooled laser removal of brain tissue at an average power of $2.7 \mathrm{~W}$, achieving an ablation speed of $2 \mathrm{~mm}^{3} \mathrm{~min}^{-1}$ and showing no major thermal damage. h, Bright-field optical image of a bovine cornea from which a flap was removed following ablation-cooled laser processing of a section $0.4 \mathrm{~mm}$ below the surface. Inset, optical coherence tomography image of the section indicated by the rectangle.

smaller by a factor of $800(2,400)$. Comparison with another reference $e^{18}$ indicates that the efficiency of ablation in our experiments is 100 times higher despite using a pulse energy that is 260 times lower, when matching the fluence of the entire burst to that of the single-pulse fluence. We achieve a level of ablation that is five times higher than results obtained with a burst-mode laser ${ }^{23}$ that does not exploit ablation cooling, despite using a pulse fluence that is 165 times smaller for the 
same burst fluence of $20 \mathrm{~J} \mathrm{~cm}^{-2}$. These results conclusively demonstrate that the exploitation of ablation cooling increases the ablation efficiency by an order of magnitude while allowing the required pulse energy to be reduced by three orders of magnitude.

We now focus on the reduction of undesired thermal effects in the ablation-cooled regime. We have performed systematic comparisons using high and low repetition rates of the same laser with identical focusing and scanning systems. Tissue removal may well be regarded as the ultimate test of the suppression of thermal effects because an increase in temperature of only a few degrees can lead to degradation. Hard-tissue experiments were conducted on human dentine to contrast the ablation-cooled regime with the traditional regime. At low average powers, the traditional regime (using $100 \mu \mathrm{J}$ pulses at $1 \mathrm{kHz}$, Fig. 3a) and the ablation-cooled regime (25 pulses of $4 \mu \mathrm{J}$ energy at a $1.7 \mathrm{GHz}$ intraburst repetition rate and $1 \mathrm{kHz}$ burst repetition rate, Fig. $3 \mathrm{~b}$ ) both provide results with negligible thermal damage (although the latter achieves an ablation rate four times higher). When increasing the processing speed by a factor of 25 with a corresponding increase in power, the traditional regime causes excessive carbonization (Fig. 3c), whereas the ablation-cooled regime does not, while achieving an ablation rate of $3 \mathrm{~mm}^{3} \mathrm{~min}^{-1}$ (Fig. 3d). Every other laser, focusing and scanning parameter was identical in these two experiments, showing that the thermal effects are greatly reduced as a result of ablation cooling.

There are numerous applications for soft-tissue ablation ${ }^{6,10,14}$, particularly in targeting the brain ${ }^{25}$, where the extreme precision afforded by a laser is of paramount importance. For this reason, we compared the effectiveness of ablation cooling in selective tissue removal from freshly harvested whole rat brains. When the average power is low, heat diffusion from the processing region to the surrounding tissue is low enough that the traditional regime avoids thermal side effects, yielding damage-free ablation ${ }^{11}$. For higher powers, the ablation-cooled regime demonstrates a clear advantage in the reduction of thermal effects: although low-repetition-rate ablation causes a broad heat-affected zone with damaged neighbouring cells, devascularization and prominent tissue loss (Fig. 3e), there is no major heat damage in the ablation-cooled regime at the same power $(600 \mathrm{~mW})$ and pulse energy $(3 \mu \mathrm{J})$ (Fig. $3 \mathrm{f})$. The corresponding ablation rate of $0.75 \mathrm{~mm}^{3} \mathrm{~min}^{-1}$ is eight times higher than when using $165 \mu \mathrm{J}, 180 \mathrm{~s}$ pulses, with which a $0.55 \mathrm{~mm}^{3}$ section of brain tissue was removed in $360 \mathrm{~s}$ (ref. 11). With ablation cooling, at a much higher power of $2.7 \mathrm{~W}(432 \mathrm{MHz}$ intraburst repetition rate, $27 \mathrm{kHz}$ burst repetition rate, $16 \mu \mathrm{J}$ per pulse), virtually thermal-damage-free results are obtained (Fig. $3 \mathrm{~g}$ ) at an ablation rate of $2 \mathrm{~mm}^{3} \mathrm{~min}^{-1}$.

Finally, we performed a flap-cutting procedure on a bovine cornea, as this is a realistic indicator for surgical applications ${ }^{8}$. An area several millimetres wide located about $0.4 \mathrm{~mm}$ below the surface of the cornea was scanned with the laser and the top layer was then lifted off with a pair of tweezers (Fig. 3h); 24 pulses with $0.8 \mu \mathrm{J}$ of energy per burst were used, which is a reduction by a factor of approximately 15 in pulse fluence compared with previous results ${ }^{8}$. This result and similar experiments on poly(methyl methacrylate) (PMMA) and hydrogels demonstrate that ablation cooling retains several of its benefits even when used for subsurface processing (see Supplementary Information section 15 for a detailed discussion).

We conclude by pointing out three speculative future directions of study: exploration of the far-from-equilibrium thermodynamics of the ablation-cooled regime, whether a suitably sculptured coherent pulse train can coherently enhance nonlinear processes ${ }^{26}$ and whether similar benefits are possible in proton therapy, because the laser-based generation of bursts of protons seems to be feasible ${ }^{27}$.

Online Content Methods, along with any additional Extended Data display items and Source Data, are available in the online version of the paper; references unique to these sections appear only in the online paper.

Received 27 July 2015; accepted 24 May 2016.

Published online 13 July; corrected online 31 August 2016

(see full-text HTML versions for details).
1. Gattass, R. R. \& Mazur, E. Femtosecond laser micromachining in transparent materials. Nat. Photon. 2, 219-225 (2008).

2. Yang, W., Kazansky, P. G. \& Svirko, Y. P. Non-reciprocal ultrafast laser writing. Nat. Photon. 2, 99-104 (2008).

3. Steinmeyer, J. D. et al. Construction of a femtosecond laser microsurgery system. Nat. Protocols 5, 395-407 (2010)

4. Plech, A., Kotaidis, V., Lorenc, M. \& Boneberg, J. Femtosecond laser near-field ablation from gold nanoparticles. Nat. Phys. 2, 44-47 (2006).

5. Rousse, A. et al. Non-thermal melting in semiconductors measured at femtosecond resolution. Nature 410, 65-68 (2001).

6. Chung, S. H. \& Mazur, E. Surgical applications of femtosecond lasers. J. Biophoton. 2, 557-572 (2009).

7. Yanik, M. F. et al. Neurosurgery: functional regeneration after laser axotomy. Nature 432, 822 (2004).

8. Juhasz, T. et al. Corneal refractive surgery with femtosecond lasers. IEEE J. Sel. Top. Quant. Electron. 5, 902-910 (1999).

9. Serbin, J., Bauer, T., Fallnich, C., Kasenbacher, A. \& Arnold, W. H. Femtosecond lasers as novel tool in dental surgery. Appl. Surf. Sci. 197-198, 737-740 (2002).

10. Hoy, C. L. et al. Clinical ultrafast laser surgery: recent advances and future directions. IEEE J. Sel. Top. Quant. Electron. 20, 242-255 (2014).

11. Loesel, F. H. et al. Non-thermal ablation of neural tissue with femtosecond laser pulses. Appl. Phys. B 66, 121-128 (1998).

12. Chichkov, B. N., Momma, C., Nolte, S., Alvensleben, F. \& Tünnermann, A. Femtosecond, picosecond and nanosecond laser ablation of solids. Appl. Phys. A 63, 109-115 (1996).

13. Bauer, F., Michalowski, A., Kiedrowski, T. \& Nolte, S. Heat accumulation in ultra-short pulsed scanning laser ablation of metals. Opt. Express 23, 1035-1039 (2015)

14. Vogel, A., Noack, J., Hüttman, G. \& Paltauf, G. Mechanisms of femtosecond laser nanosurgery of cells and tissues. Appl. Phys. B 81, 1015-1047 (2005).

15. Sutton, G. P. \& Biblarz, O. Rocket Propulsion Elements Ch. 14 (Wiley, 2011).

16. Cho, Y. I., Hartnett, J. P. \& Rohsenow, W. M. Handbook of Heat Transfer 6.21 (McGraw-Hill, 1998).

17. Hashida, M. et al. Ablation threshold dependence on pulse duration for copper Appl. Surf. Sci. 197-198, 862-867 (2002)

18. Ancona, A. et al. High speed laser drilling of metals using a high repetition rate high average power ultrafast fiber CPA system. Opt. Express 16, 8958-8968 (2008).

19. Kalaycioglu, H., Eken, K. \& Ilday, F. O. Fiber amplification of pulse bursts up to $20 \mu \mathrm{J}$ pulse energy at $1 \mathrm{kHz}$ repetition rate. Opt. Lett. 36, 3383-3385 (2011).

20. Kalaycıoğlu, H. et al. $1 \mathrm{~mJ}$ pulse bursts from a Yb-doped fiber amplifier. Opt. Lett. 37, 2586-2588 (2012).

21. Kalaycıoğlu, H., Akcaalan, O., Yavas, S., Eldeniz, Y. B. \& Ilday, F. Ö. Burst-mode Yb-doped fiber amplifier system optimized for low-repetition-rate operation. J. Opt. Soc. Am. B 32, 900-906 (2015).

22. Lapczyna, M., Chen, K. P., Herman, P. R., Tan, H. W. \& Marjoribanks, R. S. Ultra high repetition rate $(133 \mathrm{MHz})$ laser ablation of aluminum with 1.2-ps pulses. Appl. Phys. A 69, S883-S886 (1999).

23. Hu, W. Shin, Y C. \& King, G. Modeling of multi-burst mode pico-second laser ablation for improved material removal rate. Appl. Phys. A 98, 407-415 (2010)

24. Marjoribanks, R. S. et al. Ablation and thermal effects in treatment of hard and soft materials and biotissues using ultrafast-laser pulse-train bursts. Photon. Lasers Med. 1, 155-169 (2012).

25. Tsai, P. S. et al. All-optical histology using ultrashort laser pulses. Neuron 39, 27-41 (2003).

26. Meshulach, D. \& Silberberg, Y. Coherent quantum control of two-photon transitions by a femtosecond laser pulse. Nature 396, 239-242 (1998).

27. Schwoerer, H. et al. Laser-plasma acceleration of quasi-monoenergetic protons from microstructured targets. Nature 439, 445-448 (2006).

Supplementary Information is available in the online version of the paper

Acknowledgements This work was supported partially by the European Research Council (ERC) Consolidator Grant ERC-617521 NLL, the European Union (EU) FP7 CROSS TRAP and TÜBITAK under projects 112T980, 112T944 and TEYDEB-3110216. C.K. acknowledges funding from TÜBITAK - BIDEB 2211. We thank Y. Aykaç and V. Aykaç for dental experiments, T. Dalkara, M. Yemişçi Özkan, K. Kılıç for brain tissue experiments, G. Aykut for animal care and brain slicing, I. Mirza, K. Yavuz, G. Makey and M. Karatok for data acquisition and analyses, S. Karahan for histology analyses, A. Büyüksungur and BIOMATEN (METU, Ankara, Turkey) for micro-CT analyses, H. Köymen for PZT characterisation and S. Ilday, O. Tokel, H. Çelik, O. Algın and E. Atalar for critical reading of the manuscript.

Author Contributions C.K., H.K. and F.Ö.I. designed the research and interpreted the results. H.K., P.E., S.Y., Ö.A., and C.K. developed the laser systems. H.H. and R.H. developed a high-repetition-rate fibre oscillator. C.K., D.K.K. and B.Ö. performed the laser processing experiments. B.C.. and C.K. developed the numerical models. M.D.A. carried out brain slicing and histological examinations

Author Information Reprints and permissions information is available at www.nature.com/reprints. The authors declare competing financial interests: details are available in the online version of the paper. Readers are welcome to comment on the online version of the paper. Correspondence and requests for materials should be addressed to F.Ö.I. (ilday@bilkent.edu.tr).

Reviewer Information Nature thanks K. Mitra and the other anonymous reviewer(s) for their contribution to the peer review of this work. 


\section{METHODS}

The majority of the experiments were performed with a customized $\mathrm{Yb}$-doped fibre-laser, which is capable of operating in either a burst or uniform mode at a central wavelength of $1,035 \mathrm{~nm}$. This laser and the other lasers used in the experiments are detailed in Supplementary Information section 3. In burst mode, the laser produces a sequence of an adjustable number of pulses (a burst) with a high intraburst repetition rate. The bursts are repeated at a much lower repetition rate (most commonly $1 \mathrm{kHz}$ or $25 \mathrm{kHz}$ ). The intraburst repetition rate of this laser was designed to be switchable between $108 \mathrm{MHz}, 216 \mathrm{MHz}, 432 \mathrm{MHz}, 864 \mathrm{MHz}$, $1,728 \mathrm{MHz}$ and $3,456 \mathrm{MHz}$. Lower repetition rates of $1 \mathrm{MHz}$ and $27 \mathrm{MHz}$ could be obtained by selectively picking pulses using the acousto-optic modulator that is used to create the bursts. In uniform mode, the laser produced evenly spaced pulses, typically at $1 \mathrm{kHz}$ or $25 \mathrm{kHz}$. The pulse durations varied between $300 \mathrm{fs}$ and about 1 ps depending on the pulse energy. Effort was made to keep the pulse durations as similar as possible when making direct comparisons between the burst or uniform modes.

A discussion on the estimation of the minimum repetition rate for the ablationcooled regime is available in Supplementary Information section 1d. The principle criterion is for the repetition rate of the laser to be faster than the rate at which thermal energy diffuses, or is convected in case of fluids, into the surrounding regions. The dimensions of the interaction volume within which the deposited laser energy needs to be contained can be estimated as the size of the region to be ablated by the subsequent pulses, which is in the range of several hundred nanometres. For highly conductive materials, such as $\mathrm{Si}$, $\mathrm{Cu}$ or the ceramic coating of the thermocouple in Fig. 1, we estimate $\tau_{0} \approx 1$ ns. (Commonly found values for the thermal relaxation times in the scientific literature pertain to linear absorption, which is not valid for ablation by ultrafast pulses. During ultrafast ablation a plasma state is formed, which greatly changes the absorption properties.) The onset of ablation cooling is gradual (see Supplementary Figs 1 and 3) and even a repetition rate that corresponds to the inverse of $10 \tau_{0}$ confers some of the benefits of this regime. Nevertheless, we have used repetition rates that exceed $1 \mathrm{GHz}$ in most of the experiments that contrast the ablation-cooled regime with the traditional regime.

The preferred method for positioning the laser beam on the sample was to use a computer-controlled galvonometric scanner, owing to their high speeds. Alternatively, the sample can be repositioned using motorized stages. The scanning speed was adjusted such that a single burst was incident at a given spot. The laser spot size was approximately $24 \mu \mathrm{m}$ for most of the experiments. To characterize the ablation efficiency, the scanning speed was adjusted so a single pulse (in the traditional regime) or a single burst (in the ablation-cooled regime) was incident at each ablation spot to eliminate the complicated effects of crater formation and shape on the amount of material ablated. For the experiments that aimed to demonstrate a micromachining procedure, such as drilling, cutting a section of $\mathrm{Cu}, \mathrm{Si}$ or $\mathrm{PbZrTiO}_{3}(\mathrm{PZT})$ or the removal of a section of dentine, brain tissue or cornea, multiple scans were performed. This often required the readjustment of the focal plane after each layer of material had been ablated. In these experiments, the absolute durations for the completion of the process depend on the scanning and refocusing parameters. To minimize the influence of such factors, all of the parameters pertaining to the scanning procedure were kept constant when making comparisons between the traditional and ablation-cooled regimes.

Experiments that aimed to compare and contrast the ablation-cooled and traditional regimes were performed on nine different target materials: $\mathrm{Si}$ (a semiconductor), $\mathrm{Cu}$ (a metal), a thermoelectric module, PZT (a ceramic, which loses its piezoelectricity, when heated), PMMA (a transparent dielectric), dentine (a type of hard tissue) and hydrogel, brain and cornea targets (representative of soft tissues). In the case of (semi-)transparent materials, it is important to ensure that the pulse duration and peak intensities are sufficient to initiate nonlinear absorption. It is also essential that ultrafast pulses $(<10 \mathrm{ps})$ are used to avoid well-known mechanisms of thermal damage during a pulse.

The processed samples were analysed using bright-field optical microscopy, laser scanning microscopy, scanning electron microscopy and (in several cases) in situ optical coherence tomography. Histological analyses were performed used haematoxylin and eosin staining and DAPI staining procedures (Supplementary Information section 12). Soft-tissue experiments were done in accordance with the ethical standards of the Bilkent University Ethics Committee, Approval Number 2013/63. PZT, hydrogel and PMMA experiments are described in Supplementary Information sections 7, 14 and 16, respectively. Details on all of the relevant laser and scanning parameters and the target material properties used in each experiment are provided in the respective sections of Supplementary Information.

Code availability. The code used in the simulations is available on request from the corresponding author. 